\title{
Revisión y análisis documental para estado del arte: una propuesta metodológica desde el contexto de la sistematización de experiencias educativas *
}

\author{
Jorge Winston Barbosa Chacón \\ Juan Carlos Barbosa Herrera \\ Margarita Rodríguez Villabona **
}

Artículo recibido:

2 de mayo de 2013.

Artículo aceptado:

21 de mayo de 2013.

\section{Resumen}

El artículo presenta resultados de un proyecto de investigación que apuesta a realizar un estado del arte sobre la sistematización de experiencias educativas como base para resignificar y fortalecer esta estrategia en un programa académico. En esencia, se socializa el diseño y aplicación de una metodología de revisión y análisis documental (RAD) para estado del arte, la cual se caracteriza por su particular correspondencia con el problema e intereses de investigación y con la naturaleza del proceso de sistematización. Se clarifica el contexto y el diseño investigativo que demandó la realización de la RAD, pormenorizando los referentes conceptuales

* Artículo resultado del proyecto de investigación titulado: "Sistematización de experiencias en educación superior. Estado del arte de la investigación 2005-2011"; proyecto respaldado por la Vicerrectoría de Investigaciones y Extensión de la UIS con código CH2010-2. Fueron colaboradores en este proyecto: Licenciada Fanny Marcela Hormiga Sánchez y el Licenciado Juan Diego Villamizar.

** Los tres autores pertenecen a la Universidad Industrial de Santander-UIS, Colombia. (Jorge: jowins@uis.edu.co); (Juan: jbarbosa@uis.edu.co); (Margarita: marodvil@uis.edu.co).

INVESTIGACIÓN BIBLIOTECOLÓGICA, Vol. 27, Núm.61, septiembre/diciembre, 2013, México, ISSN: 0187-358X. pp. 83-105 
y procedimentales de su metodología y, en especial, la construcción de la estructura de desarrollo de la misma: fases, protocolos, etapas e instrumentos. Se emiten inferencias sobre la metodología creada en torno a: i) las razones que justifican su desarrollo; ii) la representatividad y alcance de los referentes; iii) la valoración de los elementos de su estructura de desarrollo y iv) las ventajas y escenarios de aplicación.

Palabras clave: Estado del arte; Revisión y análisis documental; Revisión sistemática de literatura; Sistematización de experiencias; Educación Universitaria.

\section{Abstract}

Documentary review and analysis for state-of-the-art assessment: a methodological proposal using educational experiences systematization approach

Jorge Winston Barbosa-Chacón, Juan Carlos Barbosa-Herrera and Margarita Rodríguez

The article presents the results of a research project aimed at creating a "state-of-the-art" approach to systematization of educational experiences as the basis for redefining and strengthening the use of educational experiences systematization within an academic program. In essence, the design and the application of a methodology for Documentary Analysis and Review (DAR) is socialized for the state-of-the-art benchmark, by establishing specific correlations between research problems and interests against nature of the systematization process. The study describes the research design and context called for by DAR, and also provides detailed definitions of theoretical, methodological and procedural sign-posts, specifically with regard to the methodology's structural development, sub-phases, protocols, stages and instruments. We draw inferences about the methodology created including: a) justification of its development; b) the representativeness and scope of its referents; c) an assessment of the components included in its developmental structure, and d) potential advantages and scenarios for its application.

Keywords: State of the Art; Documentary Analysis and Review; Systematic Literature Review; Systematization of Experiences; Higher Education. 


\section{INTRODUCCIÓN}

F ste artículo reporta parte de la experiencia investigativa de un proyecto Eadelantado por el grupo de investigación "Aprendizaje y Sociedad de la Información", ${ }^{1}$ el cual tiene al contexto universitario como escenario de participación e intervención. En tal sentido, este artículo documenta la primera acción investigativa de la apuesta por reflexionar, resignificar y darle valor agregado a la estrategia de seguimiento y observación del programa de pregrado Tecnología Empresarial, de la Universidad Industrial de Santander en Colombia. $^{2}$

En forma particular, se socializa la propuesta metodológica que respaldó la realización de una revisión y análisis documental (RAD) para un estado del arte sobre la sistematización de experiencias en contextos de educación superior como estrategia de seguimiento y observación.

Los resultados del estado del arte y la aplicación del mismo serán objeto de otra publicación. Aquí se comparte, esencialmente, "el cómo" de la RAD; es decir, su metodología específica. Ante ello el artículo se estructura en cuatro secciones que dan respuesta a interrogantes particulares:

- ¿Cuál es el contexto del proyecto? describe contextual, teórica y metodológicamente el proceso de sistematización del programa académico. Además, se describe la apuesta investigativa (el problema, los objetivos y la metodología) del proyecto en el que se circunscribe el compromiso de la RAD para el estado del arte.

- ¿Cuáles fueron los referentes conceptuales y procedimentales? pormenoriza la fundamentación que sirvió de soporte para definir la propuesta de metodología de RAD para estado del arte.

- ¿Cómo se estructuró el proceso de RAD? representa la materialización de los referentes conceptuales y procedimentales en la metodología. En ello, se detalla la estructura y aplicación de la misma en sus fases y etapas particulares.

- ¿Qué se concluye? enuncia aspectos relevantes fruto de la reflexión sobre la experiencia investigativa.

1 Grupo Aprendizaje y Sociedad de la Información. Integrado por profesores de la UIS y la Pontificia Universidad Javeriana de Bogotá. Ver: http://201.234.78.173:8080/gruplac/jsp/visuali $\mathrm{za} /$ visualizagr.jsp? $\mathrm{nro}=00000000001836$

2 Universidad Industrial de Santander. Ver: www.uis.edu.co 


\section{¿CuÁl es el Contexto del proyecto? El programa aCadémico, SU ESTRATEGIA DE SEGUIMIENTO Y EL DISEÑO INVESTIGATIVO}

Con el objeto de darle marco y justificar el compromiso de una propuesta de metodología de RAD para estado del arte, en los siguientes apartados se describe el contexto en donde se enmarca tal compromiso: el escenario educativo involucrado y su proceso de sistematización de experiencias. Posteriormente, y a la luz del anterior contexto, se describe el diseño investigativo, el cual describe la situación problema que demandó la construcción de un estado del arte sobre sistematización de experiencias y, en especial, la exigencia de un proceso particular de RAD.

Tecnología Empresarial ${ }^{3}$ es una oferta de formación de pregrado de la UIS, adscrita al Instituto de Proyección Regional y Educación a Distancia (IPRED $)^{4}$ y es desarrollada en la modalidad virtual desde 2011. El programa cuenta con un modelo pedagógico centrado en el estudiante y en el emprendimiento, y se desarrolla a través de procesos educativos caracterizados por el aprendizaje independiente, el sistema tutorial y la relación academia-sector productivo (Barbosa Chacón et al., 2010).

Los agentes educativos del programa, una vez se inició la oferta en modalidad virtual, adoptaron la sistematización de experiencias como su estrategia particular de seguimiento y observación. En ello la investigación en modalidad virtual es un referente teórico fundamental, por tener el propósito de integrar la investigación como parte integral de la práctica pedagógica (Barbosa, Rodríguez y Barbosa, 2010). Este referente, sumado a otros que respaldan el accionar de la estrategia, son descritos en el siguiente cuadro.

Cuadro 1. Enfoque conceptual de la estrategia de seguimiento y observación

\begin{tabular}{|l|l|}
\hline Elementos conceptuales & \multicolumn{1}{|c|}{ Descripción/referentes } \\
\hline Inteligencia colectiva & $\begin{array}{l}\text { Es el concepto nodal. La apuesta es lograr el mayor número de puntos de } \\
\text { vista sobre el objeto de interés: la experiencia de formación en gestión de } \\
\text { empresas (Lévy, 2004; De Kerckove, 1999). }\end{array}$ \\
\hline
\end{tabular}

3 Tecnología Empresarial es un programa del Instituto de Proyección Regional y Educación a Distancia, IPRED, de la UIS, que busca formar un profesional integral con conocimientos en el área de Gestión de Empresas, fundamentado en el desarrollo de una mentalidad emprendedora, con capacidad para comprender e interpretar la realidad del entorno, para liderar procesos de cambio en la modernización de las empresas y organizaciones ya establecidas y/o creador de empresa por el progreso y mejor calidad de vida de la sociedad. Ver: http://ead.uis. edu.co/empresarial/y http://ead.uis.edu.co/emprendedores/

4 Ver: http://www.uis.edu.co/webUIS/es/academia/institutoProyeccionRegionalyEducacionDis tancia/index.html 


\begin{tabular}{|c|c|}
\hline Construcción colectiva & $\begin{array}{l}\text { Se genera a través del aprendizaje sobre la experiencia y toma como base } \\
\text { los diferentes puntos de vista sobre las situaciones creadas en la conjunción } \\
\text { de condiciones curriculares (plan de estudios, prácticas docentes, interac- } \\
\text { ciones, la metodología, la dinámica organizacional y el uso de recursos tec- } \\
\text { nológicos) (Jiménez y Calzadilla, 2011; Pontual y Price, 2010). }\end{array}$ \\
\hline Visión sociocultural y crítica & $\begin{array}{l}\text { Se materializa en el interés de los agentes educativos por la experiencia mis- } \\
\text { ma. El proceso respalda el papel de la cultura en el aprendizaje y el conoci- } \\
\text { miento como un producto histórico de producción colectiva. De allí se deriva } \\
\text { que, para comprender la dinámica del ambiente de aprendizaje diseñado, es } \\
\text { necesario trabajar con los diferentes agentes educativos que participan en } \\
\text { él y crear las condiciones para que sus puntos de vista influyan en las deci- } \\
\text { siones (Lévy, 1999; Bruner, 1988; Morín, 1999; Wertsch, 1988; Wenger, } \\
\text { McDermott y Snyder, 2002). }\end{array}$ \\
\hline $\begin{array}{l}\text { Zona proximal de desarrollo, } \\
\text { andamiaje, apropiación y } \\
\text { mediación }\end{array}$ & $\begin{array}{l}\text { Son base para orientar las decisiones relativas a los elementos presentes } \\
\text { en el ambiente de aprendizaje (agentes educativos y procesos) y permiten } \\
\text { dimensionar los alcances de la transformación continua que se hace en el } \\
\text { programa (Pérez Alcalá, 2009; Padilla Partida, 2006; Guk y Kellogg, 2007). }\end{array}$ \\
\hline $\begin{array}{l}\text { Aprendizaje situado y } \\
\text { comunidades de práctica }\end{array}$ & $\begin{array}{l}\text { Importantes para repensar el papel de los tutores, de los recursos de apoyo } \\
\text { al aprendizaje y la dinámica de trabajo colaborativo que sirve de marco me- } \\
\text { todológico para la planeación de las producciones (Wertsch, 1988; Wenger, } \\
\text { McDermott y Snyder, 2002). }\end{array}$ \\
\hline Virtualización & $\begin{array}{l}\text { Concebida como proceso de transformación en flujo constante y como } \\
\text { movimiento entre la identificación de los aspectos fundamentales de las } \\
\text { experiencias educativas, su problematización, su desterritorialización, la } \\
\text { comprensión de sus flujos entre lo exterior y lo interior, entre lo público y lo } \\
\text { privado (Lévy, 1999; Lévy, 2004; Bauman, 1999). }\end{array}$ \\
\hline Las tecnologías & $\begin{array}{l}\text { Se comprende que las tecnologías son el resultado de un conjunto de situa- } \\
\text { ciones concretas que obligan a mantener una actitud crítica sobre ellas y } \\
\text { el impacto de su incorporación en los procesos educativos, y su respuesta } \\
\text { a necesidades específicas de aprendizaje (Postman, 1996; Sígales, 2004; } \\
\text { García-Valcárcel y Tejedor, 2010; Youssef y Dahmani, 2008). }\end{array}$ \\
\hline
\end{tabular}

Fuente: basado en Barbosa, Rodríguez y Barbosa (2010).

Desde lo conceptual, la sistematización de experiencias educativas es un proceso que responde a la fundamentación teórica que soporta la investigación en educación desde el paradigma cualitativo, buscando explorar contextos y sujetos para lograr descripciones y explicar la realidad subjetiva de la acción (Restrepo y Tabares, 2000).

En correspondencia, la sistematización ha sido entendida y abordada como un camino de recuperación, análisis y apropiación de las prácticas educativas del programa académico que, al relacionar sistémica e históricamente sus componentes teóricos y prácticos, permite comprender y explicar los contextos, el sentido, los fundamentos, las lógicas y los aspectos problemáticos que presentan tales prácticas (Ruiz citando a Ghiso, 2001). De esta manera, los agentes educativos han adoptado la concepción de sistematización como mirada de saberes propios sobre la práctica (Barbosa, Rodríguez y Barbosa, 2010). 
Metodológicamente, y desde una concepción metacognitiva, cada uno de los agentes educativos se compromete con el registro de su propia experiencia. Esto exige la expresión de sus ideas, pensamientos o emociones sobre la experiencia de formación, tal cual como la viven. Ese registro es objeto de reflexión al que se le añaden elementos contextuales que comprenden los hechos; insumos para la toma de decisiones e intervención.

La toma de decisiones e intervención se centra en los ejes nucleares de la propuesta formativa: i) la metodología comprende los procesos y los diferentes roles de los agentes educativos y ii) los guiones de aprendizaje como la principal herramienta de acompañamiento. Están así pensados, organizados e integrados los elementos que componen la ruta de aprendizaje de una asignatura en particular que sirve como camino de navegación y regla de juego de un curso (Barbosa, Rodríguez y Barbosa, 2010; Barbosa, 2010).

Con el objeto de describir procedimentalmente la estrategia, en el siguiente cuadro se hace una descripción general de los participantes y de las fases de desarrollo.

Cuadro 2. Elementos base de la estrategia de seguimiento y observación

\begin{tabular}{|l|l|}
\hline Elementos base & \multicolumn{1}{|c|}{ Descripción } \\
\hline Participantes & $\begin{array}{l}\text { Son agentes educativos participantes: i) los estudiantes activos; ii) los estudiantes orien- } \\
\text { tadores (apoyan el desarrollo de los cursos); iii) los tutores (líderes y de asignatura); iv) } \\
\text { el grupo base (líderes de las dimensiones pedagógica, formación, producción y soporte } \\
\text { tecnológico) y el grupo líder de sistematización. }\end{array}$ \\
\hline Registro & $\begin{array}{l}\text { Son bases documentales de la experiencia: i) el diario de participantes; ii) la actividad de } \\
\text { cada uno de los cursos en línea; iii) las memorias de: colectivos de tutores, reuniones del } \\
\text { grupo base y conversaciones con los estudiantes. }\end{array}$ \\
\hline Análisis & $\begin{array}{l}\text { Momentos de análisis individuales: i) el reporte individual de la experiencia. Este reporte } \\
\text { se basa en los registros del diario de la experiencia, utilizando los elementos del guión } \\
\text { de aprendizaje como categorías de análisis y ii) la elaboración del reporte consolidado de } \\
\text { cada punto de vista, a cargo de los tutores líderes de asignatura. } \\
\text { Momentos de análisis grupales: i) la discusión grupal de los reportes consolidados; ii) la } \\
\text { generación de categorías que permiten analizar el corpus textual derivado de todo el tra- } \\
\text { bajo y iii) el análisis cualitativo de todos los registros para lo cual se hace uso de software. }\end{array}$ \\
\hline
\end{tabular}

Fuente: basado en Barbosa, Rodríguez y Barbosa (2010).

Dada la representatividad que tiene la dinámica de la sistematización en el programa académico, y siendo consciente de la necesidad del fortalecimiento de la misma, surgió el compromiso de contar con un cimiento que permitiera construir una mirada más sólida sobre esta estrategia, dadas sus implicaciones en el desarrollo, la investigación y la calidad del programa. Por ello se tomó la decisión de repensar la estrategia, tomando como base el desarrollo de un estado del arte sobre procesos de sistematización en otros 
escenarios; compromiso que permitiera responder a la pregunta ¿cuál es el estado actual de la sistematización de experiencias en Educación Superior y cómo a partir de él se puede fortalecer esta estrategia en el programa académico?

Para el logro del anterior compromiso se generaron objetivos específicos encaminados a realizar una consulta y una categorización de fuentes de información relacionadas con proyectos y experiencias de sistematización en contextos universitarios; a analizar la información desde una perspectiva crítica que permitiera dilucidar la dinámica y lógica de la fundamentación teórica y metodológica de los procesos de sistematización y, finalmente, a formular planteamientos que, a partir de las comprensiones y construcciones de lo investigado, estuvieran encaminados a resignificar y fortalecer la estrategia de seguimiento y observación.

Con el problema y los objetivos definidos, se asumió un compromiso por observar una metodología sistemática para el tratamiento de fuentes de información, la cual ofreciera una estrategia organizada y coherente de búsqueda y análisis, en favor de la realización de una síntesis crítica. En concordancia, construir una metodología particular de RAD para el estado del arte se proyectó como el insumo base para afrontar el compromiso investigativo circunscrito, en donde los procesos de interpretación, crítica y argumentación fueran los pilares para facilitar la creación de inferencias y relaciones (Delgado y Forero, 2004).

En correspondencia, el proceso de concreción de los dos primeros objetivos específicos y, en particular, el desarrollo de una metodología de RAD para el estado del arte, son la base de todo lo que se socializa a continuación.

\section{¿CUÁLES FUERON LOS REFERENTES CONCEPTUALES Y PROCEDIMENTALES? LA FUNDAMENTACIÓN QUE SOPORTÓ LA METODOLOGÍA DE RAD}

La fundamentación que respaldó la metodología de RAD condujo a procesos de resignificación y apropiación que, a la luz del diseño de investigación, permitieron definir la propuesta metodológica respectiva.

Conceptualmente se tuvieron en cuenta los fundamentos que respaldan el desarrollo de los estados del arte como investigación documental; investigación desde la que se obtiene y se trasciende el conocimiento acumulado en torno a un objeto de estudio en un momento determinado (Posada, 2011; Gutiérrez, 2012; Vélez y Galeano, 2002; Castañeda, 2004). Estado del arte es investigar desde la óptica de "ir tras las huellas" del campo de conocimiento que se pretende ahondar, permitiendo determinar cómo ha sido tratado éste y cuáles son las tendencias (Rojas Rojas, 2007; Caro Gutiérrez et al., 2005) 
pero también hay que asumirlo como una investigación sobre la producción investigativa, teórica o metodológica, en donde emerge la posibilidad de articular las conceptualizaciones, discursos y prácticas, así como indagar por la dinámica y lógica de dicha producción (Uribe, 2004; Gutiérrez, 2012). Por todo esto, un estado del arte no es un producto terminado, ya que es un insumo a nuevos campos de investigación (Castañeda, 2004; Hoyos, 2000).

Como investigación documental, el estado del arte es orientado por varios principios que dan rigor a su desarrollo, así: i) Finalidad. Representa el compromiso por establecer objetivos de investigación previos; ii) Coherencia. Es contar con unidad interna en materia de fases, actividades y datos; iii) $F i$ delidad. Alude a un respaldo en materia de recolección y transcripción; iv) Integración. Implica articulación y evaluación global del proceso y iv) Comprensión. Se traduce en el favorecimiento de la construcción teórica sobre el objeto de estudio (Hoyos, 2000; Castañeda, 2004).

En el caso del trabajo adelantado, se asumió la postura de "ir tras las huellas" del conocimiento para apoyar la solución de un problema de investigación definido (Budgen y Brereton, 2006). Ello implica definir con claridad qué huellas seguir y cómo ir tras ellas (Carro, 2008; Rojas Rojas, 2007); compromisos que, incluso, permiten trazar las huellas del futuro en cuanto a investigación se refiere (Londoño, 2007; Caro Gutiérrez et al., 2005).

Definir "qué huellas seguir" implica un compromiso con el rigor de la consulta y revisión. Al respecto, se centró la mirada sobre experiencias de investigación que tuvieran la mayor cercanía con el problema de investigación. Para el caso, representó un esfuerzo por hacer investigación de la investigación (Pantoja, 2006); esfuerzo que contribuye a generar nuevo conocimiento y favorece la elaboración de producciones propias (Agudelo, Santana y Velandia, 2005).

En consecuencia, y bajo la apuesta de hacer un estado del arte en donde su RAD contara con un sendero metodológico expedito, resultó pertinente seguir las orientaciones de Arellano y Santoyo (2012), quienes proponen trazar un trabajo con un horizonte claro a manera de tríada: estado de las fuentes, estado de la información y estado del conocimiento.

De cara a lo procedimental y, en particular, para responder a la pregunta ¿cómo ir tras las huellas? se tomaron como base los fundamentos de estados del arte en cuanto a sus fases de desarrollo macro: heurística o de recolección de fuentes de información y hermenéutica o de análisis.

La fase heurística, de orden preparatorio, representa el procedimiento de búsqueda y recopilación de fuentes de información según su naturaleza y características; es la aproximación al objeto de estudio, a través de la delimitación y definición de estrategias particulares de búsqueda (Rojas Rojas, 
2007; Hoyos, 2000; Bucheli y Córdoba, s.f.; Lopera y Adarve, 2008; Castañeda, 2004).

Para un desarrollo efectivo de esta primera fase, se recomienda la planificación y desarrollo de protocolos de búsqueda y revisión de fuentes de información, como estrategia que favorece un desarrollo coherente y contextualizado (Budgen y Brereton, 2006; Caro Gutiérrez et al., 2005). De igual manera, y para favorecer el desarrollo del protocolo de revisión de fuentes de información, en particular la estrategia de extracción de datos, se tuvo en cuenta la sugerencia de construir una ficha de recolección de datos como instrumento que apoya el filtrado de información de interés específico (Hoyos, 2000; Castañeda, 2004; Fernández, 2008).

La fase hermenéutica representa el trabajo de lectura, análisis, interpretación y comprensión crítica y objetiva en concordancia con los propósitos de investigación; permite ampliar marcos de referencia sobre el estudio en particular y es donde se realiza un trabajo crítico que señala identidad; es también actividad de reflexión que permite una captación de sentido en los textos en diferentes contextos (Rojas y Rojas, 2007; Bucheli y Córdoba, s.f.; Hoyos, 2000; Posada, 2011; Duarte, Zapata y Rentería, 2010; Fernández, 2008; Castañeda, 2004).

Es importante destacar que las fases antes descritas tienen correspondencia con las líneas relacionadas con las maneras de generar nuevo conocimiento: la visión heurística y la visión hermenéutica; aspecto descrito por Bosh (s.f.). Desde sus planteamientos la visión heurística: i) se apoya en principios con los cuales se realizan búsquedas conducentes a avanzar en el conocimiento; ii) parte de ideas fundantes que son la base para codificar lo conocido y iii) es la posibilidad de ampliar o mostrar lo conocido, creando los fundamentos del nuevo conocimiento en relación con lo ya conocido.

En cuanto a la visión hermenéutica, el mismo autor acota: i) se centra en procesos de análisis e interpretación de fuentes de conocimiento; ii) desde su enfoque, la construcción de conocimiento surge de forma interpretativa, sectorial, no dogmática, y priman los marcos de referencia generados por otras prácticas hermenéuticas.

De manera complementaria y al identificarse como un elemento sustancial para apoyar el desarrollo de la fase heurística, se dio crédito a orientaciones metodológicas sobre el desarrollo de revisiones sistemáticas de literatura, que son entendidas como investigaciones científicas en sí mismas, con métodos prefigurados y un ensamblaje en torno a estudios originales (Gisbert y Bonfill, 2004; Caro Gutiérrez et al., 2005). De igual forma, son entendidas como una manera de valorar e interpretar investigaciones disponibles y relevantes en relación con una interrogante de investigación particular (Kitchenham, 2004). 
Como referente preliminar para desarrollar la fase hermenéutica, se dio importancia al interés de análisis e interpretación del grupo investigador: develar la dinámica y lógica tanto de los diseños de investigación como de la teorización y procedimientos particulares de la sistematización de experiencias educativas.

Para responder a este interés el desarrollo de la fase hermenéutica tomó como base los fundamentos del enfoque de análisis cualitativo crítico que, como estrategia posicionada, le ha dado a la "categorización" el nivel que le corresponde en la investigación. En correspondencia, el proceso de análisis se soportó en los planteamientos de Strauss y Corbin (2002) y Glaser y Strauss (1967), en particular, planteamientos sobre la teoría fundada; teoría que permite contextualizar y desvelar las interacciones y construcciones que se hacen sobre una actividad, así como sus componentes culturales (De la Cuesta, 2006). Cabe reconocer que esta teoría tiene especial aplicación en el análisis cualitativo de documentos que puede incluir el apoyo de herramienta software.

\section{¿CÓMO SE ESTRUCTURÓ EL PROCESO DE RAD? \\ De los REFERENTES A LA METODOLOGÍA}

La metodología de la RAD se concretó a partir de la reflexión, adaptación y aplicación de los referentes conceptuales y procedimentales, de lo cual emergieron las estructuras, elementos e instrumentos asociados a sus fases de desarrollo: heurística y hermenéutica.

Desde lo logístico, y obrando en correspondencia con la dinámica colectiva de la estrategia de seguimiento y observación, inicialmente descrita, la generación de la propuesta metodológica de RAD, y su respectiva aplicación, fue una responsabilidad colectiva entre el equipo líder de sistematización (tres investigadores con experiencia en sistematización, educación mediada por TIC y formación en gestión de empresa); una experta en lengua extranjera (inglés) y un investigador social con dominio de software para el análisis cualitativo.

\section{Fase heurística}

Para su desarrollo se diseñó una guía de fase heurística, la cual permitió visualizar las etapas y acciones pertinentes con el desarrollo de esta fase; guía que se estructuró a través de protocolos de búsqueda y revisión de fuentes de información. 
El primer protocolo se estructuró en cinco elementos: i) el idioma de las fuentes de información; ii) el periodo de tiempo; iii) la generación de los términos de búsqueda; iv) los recursos posibles de provisión de fuentes de información y v) la propuesta de estrategias particulares para la búsqueda de dichas fuentes. Por su parte, el segundo protocolo se estructuró en cuatro elementos: i) las normas particulares de revisión; ii) los criterios de exclusión (determinados a partir del problema y los intereses del grupo investigador); iii) los criterios de inclusión representados en los tópicos relevantes a la investigación y iv) la estrategia de extracción de datos. En el Cuadro 3 se aprecia la guía de fase heurística, mostrando aspectos directos de su aplicación.

Cuadro 3. Guía de desarrollo de fase heurística

\begin{tabular}{|c|c|c|}
\hline \multicolumn{3}{|c|}{ A. Protocolo de búsqueda de fuentes de información } \\
\hline & Idioma & Español e inglés \\
\hline & Periodo de tiempo & $2005-2011$ \\
\hline \multirow[t]{2}{*}{ Términos } & Individuales & $\begin{array}{l}\text { Sistematización; Educación; Experiencias; Estrategias; Seguimiento; } \\
\text { Observación; Dialéctica; Didáctica; Metodología; Práctica; Reflexión; } \\
\text { Aprendizaje; Investigación; Investigación-acción; Inteligencia colectiva; } \\
\text { Construcción colectiva; Aprendizaje situado; Virtualización; Educación } \\
\text { superior. } \\
\text { Action research; Education; Case study; Transparency; Transferability; } \\
\text { Analytical generalization; Learning; Collaboration; Community of practice; } \\
\text { Constructivism; Group work; Interaction; Participatory research. }\end{array}$ \\
\hline & Combinación & $\begin{array}{l}\text { Sistematización de experiencias educativas; Visión sociocultural y críti- } \\
\text { ca; Movimientos sociales; Investigación participativa; Memoria y narrati- } \\
\text { vas; Conocimiento fuente, Conocimiento meta; Suscitación de la poten- } \\
\text { cia deliberativa; Estilos de aprendizaje, Enfoque de aprendizaje; Learning } \\
\text { Styles; Learning Approach. }\end{array}$ \\
\hline $\begin{array}{l}\text { Recursos de } \\
\text { información }\end{array}$ & \multicolumn{2}{|c|}{$\begin{array}{l}\text { i) Bases de datos: Ebscot Host, Science Sliever, Proquest, ISI Web of Knowledge, Dialnet, } \\
\text { Redalyc; ii) Google Académico; iii) revistas indizadas en formato digital (educación, tra- } \\
\text { bajo social); iii) memorias de eventos académicos y específicos sobre sistematización de } \\
\text { experiencias; iv) páginas de redes sobre sistematización de experiencias; v) trabajos de } \\
\text { investigación de posgrado. }\end{array}$} \\
\hline \multirow[t]{3}{*}{ Estrategias } & De formación & Asesoría en uso de bases de datos, en función de los intereses de consulta. \\
\hline & \begin{tabular}{|l|} 
De generación \\
de términos
\end{tabular} & Combinación entre revisión de títulos y resúmenes de artículos. \\
\hline & De búsqueda & $\begin{array}{l}\text { i) Ingreso escalonado de términos, restringiendo la búsqueda según } \\
\text { resultado precedentes; ii) búsquedas booleanas (sencillas y por ecua- } \\
\text { ciones); iii) consulta a páginas personales de expertos y iv) revisión de } \\
\text { citaciones y referencias bibliográficas. }\end{array}$ \\
\hline \multicolumn{3}{|r|}{ B. Protocolo de revisión de fuentes de información } \\
\hline $\begin{array}{l}\text { Normas de } \\
\text { revisión }\end{array}$ & \multicolumn{2}{|c|}{$\begin{array}{l}\text { - Diagnosticar la existencia de trabajos análogos o cercanos sobre el objeto de estudio. Para } \\
\text { el caso: sistematización de experiencias, action researchy capitalización de experiencias. } \\
\text { - Revisar las fuentes de información teniendo al horizonte de investigación (problema y } \\
\text { objetivos) como referente constante de revisión. } \\
\text { - Corroborar la correspondencia de la fecha de publicación de la fuente primaria. }\end{array}$} \\
\hline
\end{tabular}




\begin{tabular}{|c|c|c|}
\hline \multirow{2}{*}{\multicolumn{2}{|c|}{\begin{tabular}{|l} 
\\
$\begin{array}{l}\text { Criterios de } \\
\text { exclusión }\end{array}$
\end{tabular}}} & $\begin{array}{l}\text { - Corroborar y conseguir los trabajos completos, constatando su relevancia para la investigación. } \\
\text { - Hacer lectura del resumen e introducción como estrategia de inclusión o exclusión preliminar. } \\
\text { - Hacer registro de comentarios acerca del estudio (relevancia, aspectos destacados o } \\
\text { cualquier otro aspecto útil). }\end{array}$ \\
\hline & & $\begin{array}{l}\text { - Publicaciones que no contengan información de interés a pesar de contener los términos } \\
\text { de búsqueda o combinación de ellos. } \\
\text { - Publicaciones relacionadas con experiencias cuyos contextos de intervención no son educativos. } \\
\text { - Publicaciones relacionadas con experiencias cuyos diseños investigativos distan radicalmen- } \\
\text { te del enfoque de sistematización de experiencias adoptado por el programa académico. }\end{array}$ \\
\hline & $\begin{array}{l}\text { Criterios de } \\
\text { inclusión }\end{array}$ & $\begin{array}{l}\text { Los tópicos relevantes surgieron de dos procesos particulares: } \\
\text { - De la visión del grupo líder de sistematización de cara al abordaje del problema. Fueron } \\
\text { tópicos: i) los referentes teóricos y procedimentales de la sistematización; ii) los roles de } \\
\text { los participantes y iii) la manera como se construye y aplica el conocimiento. } \\
\text { - De los elementos considerados como ejes en una experiencia de sistematización, los } \\
\text { cuales fueron deducidos a partir de los planteamientos de dos expertos que han sido } \\
\text { referencia conceptual y procedimental para el grupo líder de sistematización. Fueron } \\
\text { tópicos: i) el análisis, la deconstrucción, la interpretación, la articulación y la referencia- } \\
\text { ción de las prácticas (se narran, describen y relatan) y las percepciones (se reconocen, } \\
\text { develan y comunican) de la experiencia (Ghiso, 2008); ii) las concepciones de sistemati- } \\
\text { zación, las rutas propuestas, los roles de los agentes, el diseño y el uso de instrumentos } \\
\text { y medios para registro, la interpretación, la construcción de categorías y la producción de } \\
\text { conocimiento (Mejía, 2008). }\end{array}$ \\
\hline & $\begin{array}{l}\text { Estrategia de } \\
\text { extracción de } \\
\text { datos }\end{array}$ & $\begin{array}{l}\text { Representó el diseño del formato de revisión documental, el cual se estructuró en tres sec- } \\
\text { ciones: i) datos de diligenciamiento; ii) datos descriptivos de la publicación y iii) tópicos } \\
\text { relevantes de la publicación. Este formato fue una construcción colaborativa entre los inte- } \\
\text { grantes del grupo líder en sistematización y fue sometido a un piloto con fuentes primarias. } \\
\text { Posteriormente, y a medida que se adelantó la revisión, el formato fue objeto de fortaleci- } \\
\text { miento y ajuste en correspondencia con los hallazgos. }\end{array}$ \\
\hline & & C. Valoración de la fase heurística \\
\hline & $\begin{array}{l}\text { La planificació } \\
\text { valoración y fo } \\
\text { intereses de in }\end{array}$ & $\begin{array}{l}\text { desarrollo de esta fase y, en particular, el diseño y aplicación de la guía, fueron objeto de } \\
\text { lecimiento continuo a cargo del equipo responsable de la RAD, a la luz del problema y de los } \\
\text { tigación (criterios de inclusión). }\end{array}$ \\
\hline
\end{tabular}

Fuente: basado en Kitchenham (2004), Budgen y Brereton (2006) y Caro Gutiérrez et al. (2005).

Adicionalmente y para dar cuenta del desarrollo del protocolo de revisión de fuentes de información se construyó una ficha de recolección; instrumento que fue denominado formato de revisión documental, el cual dio cuenta de los criterios de inclusión y los tópicos relevantes para la investigación, los cuales se constituyeron en objeto de rescate en las fuentes de información.

A continuación se puede apreciar la estructura del formato de revisión documental, el cual, para su entendimiento, muestra los descriptores de los tópicos relevantes, los cuales fueron descritos en el protocolo de revisión de la guía de la fase heurística. 
REVISIÓN Y ANÁLISIS DOCUMENTAL PARA ESTADO DEL ARTE:...

Cuadro 4. Formato de revisión documental

\begin{tabular}{|c|c|c|}
\hline \multicolumn{3}{|c|}{$\begin{array}{l}\text { Formato de revisión documental } \\
\text { Fundamentación teórica y metodológica de sistematización } \\
\text { de experiencias en educación universitaria }\end{array}$} \\
\hline \multicolumn{3}{|c|}{ Datos de diligenciamiento } \\
\hline \multicolumn{3}{|l|}{ Núm. de guía } \\
\hline \multicolumn{3}{|l|}{ Fecha de diligenciamiento } \\
\hline \multicolumn{3}{|l|}{ Elaborada por } \\
\hline \multicolumn{3}{|l|}{$\begin{array}{l}\text { Tiempo utilizado en el } \\
\text { diligenciamiento }\end{array}$} \\
\hline \multicolumn{3}{|c|}{ Datos descriptivos de la publicación } \\
\hline \multicolumn{3}{|l|}{ Título de la publicación } \\
\hline \multicolumn{3}{|l|}{ Fecha de publicación } \\
\hline \multicolumn{3}{|l|}{ Título del proyecto } \\
\hline \multicolumn{3}{|l|}{ Autor/es } \\
\hline \multicolumn{3}{|l|}{ Palabras clave } \\
\hline \multicolumn{3}{|l|}{ Lugar de creación } \\
\hline \multicolumn{3}{|c|}{ Tópicos relevantes de la publicación } \\
\hline \multirow[t]{2}{*}{ Descripción general } & Objetivo/s & \\
\hline & Justificación & Descripción del para qué del proceso de sistematización. \\
\hline \multirow[t]{2}{*}{ Fundamentación teórica } & $\begin{array}{l}\text { Concepción(es)/ } \\
\text { concepto(s) de siste- } \\
\text { matización }\end{array}$ & $\begin{array}{l}\text { Elemento orientador de la sistematización. Ejemplos: sis- } \\
\text { tematización como: i) una recuperación de la experiencia } \\
\text { en la práctica; ii) como producción de conocimiento; iii) } \\
\text { como forma de empoderar los sujetos de la práctica; iv) } \\
\text { como investigación social y v) otro. }\end{array}$ \\
\hline & Enfoque(s) & $\begin{array}{l}\text { Respaldo epistemológico del proceso de sistematización } \\
\text { (histórico-dialéctico, dialógico interactivo, deconstructi- } \\
\text { vo, reflexividad y construcción de la experiencia humana } \\
\text { y enfoque hermenéutico). }\end{array}$ \\
\hline \multirow{6}{*}{$\begin{array}{l}\text { Metodología del proce- } \\
\text { so de sistematización }\end{array}$} & Fases & Descripción de las etapas del proceso de sistematización. \\
\hline & Actividades & $\begin{array}{l}\text { Conjuntos de tareas u operaciones de las fases del pro- } \\
\text { ceso de sistematización. }\end{array}$ \\
\hline & Población participante & Actores líderes y participantes (internos y externos). \\
\hline & Recursos & Conjunto de elementos tecnológicos, físicos y otros. \\
\hline & $\begin{array}{l}\text { Mecanismos para la } \\
\text { generación de fuentes } \\
\text { de información }\end{array}$ & $\begin{array}{l}\text { Descripción de mecanismos para recoger información de } \\
\text { prácticas y precepciones (instrumentos, técnicas, talle- } \\
\text { res, conversatorios, otros). }\end{array}$ \\
\hline & $\begin{array}{l}\text { Análisis e interpre- } \\
\text { tación }\end{array}$ & $\begin{array}{l}\text { Descripción del proceso de análisis de información (el } \\
\text { cómo del análisis de la información). Puede incluir as- } \\
\text { pectos como la generación metodológica de categorías y } \\
\text { el tratamiento a interrogantes y supuestos. }\end{array}$ \\
\hline \multicolumn{2}{|c|}{ Conclusiones/discusión final } & $\begin{array}{l}\text { Es otro tipo de análisis, entendido como las reflexiones fi- } \\
\text { nales en torno a la experiencia vivida. Puede verse como el } \\
\text { contraste final que se hace entre los objetivos y la vivencia; } \\
\text { puede hacer referencia al impacto logrado y, en algunos } \\
\text { casos, a las relaciones con otros proyectos análogos. }\end{array}$ \\
\hline
\end{tabular}




\begin{tabular}{|l|l|}
\hline Sistematización de la sistematización & $\begin{array}{l}\text { Responde a discusiones sobre el proceso metodológico } \\
\text { efectuado y a vivenciar lo que no es sistematización. }\end{array}$ \\
\hline Referentes base & Referentes teóricos y metodológicos estructurales. \\
\hline Observaciones & Precisiones, adicionales y otros. \\
\hline
\end{tabular}

Fuente: construcción propia.

\section{Fase hermenéutica}

El desarrollo de esta fase, al igual que la anterior, tuvo como base el problema de investigación y, en especial, el compromiso de análisis e interpretación trazado por el grupo líder de sistematización, el cual fue manifiesto en los referentes.

En particular el concepto de análisis asumido hizo referencia a un acto mental de distinción y separación de las partes de un "todo" con el objeto de conocer sus elementos definitorios. De esta manera el análisis fue enmarcado en un procedimiento particular: i) ordenamiento y manipulación de la información en sus fuentes y resumen de datos; ii) escritura detallada de categorías y relaciones entre éstas; iii) refinamiento de la búsqueda de categorías centrales y iv) texto analítico final. Para el desarrollo de este procedimiento, en particular para el procesamiento de la información, se utilizó el software ATLAS.ti.

A continuación se pormenorizan las siete etapas que enmarcan el proceso de análisis adelantado:

1. Gestión documental para el análisis. Con el propósito de obtener una compresión de los saberes y significados del objeto de estudio implícito en la información recolectada, se creó una unidad hermenéutica que contuvo el cuerpo documental, el cual fue organizado y sometido a un proceso de reflexión crítica, a través de un análisis por comparación constante.

2. Estructuración conceptual previa. Se identificaron unos patrones conceptuales que emergieron de los principales elementos del proyecto de investigación (contexto, diseño investigativo y referentes conceptuales y procedimentales e interés de análisis particular). La detección de esos patrones cumplió el papel de ser el referente para el proceso de segmentación de descriptores, descrito más adelante.

3. Creación de categorías núcleo. Desde estas categorías surgió el proceso de taxonomía o niveles de categorización: el primer nivel corresponde a las categorías núcleo, el segundo a las subcategorías y el tercero a las sub-subcategorías. 
Como se apreciará, esta etapa conectó sistémicamente actividades de análisis posteriores como la segmentación de la información, la creación y definición de categorías, la relación categorial y la creación de memos analíticos sobre hallazgos en la información; todo esto desde una postura de flexibilidad analítica.

4. Segmentación de la información automatizada. ATLAS.ti permitió facilitar la segmentación por descriptores y, de esta manera, generar una codificación automática respaldada en una verificación manual de los resultados, lo que posibilitó un análisis con calidad y pertinencia conceptual.

La codificación automática se respaldó en la búsqueda de descriptores que hace el software por medio de una cadena de palabras que tienen una relación semántica con una categoría. Dicha cadena se elabora por medio de la agrupación de palabras de una lista que arroja la herramienta, generando un reporte de cada palabra con su respectiva frecuencia dentro de los documentos. Con ese insumo se fueron distribuyendo dichas palabras hacia las categorías núcleos, sirviendo como patrones para agrupar.

5. Identificación de descriptores. Se pusieron en funcionamiento las agrupaciones de palabras para obtener los descriptores correspondientes (citas de los textos) para posteriormente realizar un proceso de refinamiento a la búsqueda automática de los mismos. Este refinamiento consistió en reagrupar adecuadamente los descriptores con respecto a la categoría núcleo a la que pertenecían.

6. Categorización emergente. Partiendo de los respectivos descriptores de cada categoría núcleo, se generaron las categorías emergentes (subcategorías y sub-subcategorías). El proceso de categorización se estructuró en dos ámbitos: descriptivo y teórico. Al primero correspondieron todas las categorías donde se identificaron patrones, regularidades, inconsistencias, discontinuidades, principios, incoherencias, categorías de contexto, categorías de escenarios, categorías de perspectiva de participantes y sus relaciones, categorías de proceso y categorías de estrategias. Estas categorías descriptivas sirvieron para abrir el análisis y optimizarlo en las situaciones de dudas de la selección de la categoría más adecuada para el descriptor que se estaba trabajando. El ámbito teórico se creó partiendo de las categorías descriptivas para dar el salto a categorías más abstractas y relacionales. Esas últimas se construyeron desde la teoría y se relacionaron con dos o más categorías a su cargo.

Los subniveles de las categorías se construyeron reflexionando sobre sus propiedades (componentes del objeto de análisis) y dimensiones (cualidades de los componentes del objeto de análisis). Se definió cada 
subcategoría para entenderla dentro del contexto particular que se estaba reflexionando.

A la par de las definiciones de cada categoría, se construyeron memos analíticos donde se iban consignando las ideas que emergían con la lectura de la información. Los memos se construyeron con base en tres elementos: i) registro del grupo investigador sobre la idea o el hallazgo que se tiene (creación de nuevas categorías, modificación o aparición de nuevos supuestos); ii) registro de lo que arroja el descriptor y iii) el registro de la conexión de los hallazgos (categorías) con la teoría formal existente.

7. Mapeo de conexiones y elaboración textual. A partir de la categorización se exportaron los resultados del proceso de análisis en matrices cualitativas; teniendo como hallazgos los descriptores que se interpretaron a partir de las categorías.

A continuación se hizo el análisis, identificando conexiones generales entre categorías. Con estas conexiones se hicieron tres tipos de textos: i) texto representado por los memos analíticos presentes en la matriz; ii) texto que integra los memos, en él se inicia la elaboración de conexiones entre las categorías centrales que se han identificado, se reformulan y se especifican sus dimensiones. Con este segundo texto se vuelve sobre los datos en una actividad de contrastación, de tal manera que se afinen las conexiones. Para realizar esta tarea se recurre al mapeo del texto inicial y la identificación de nuevos elementos de análisis de los enunciados existentes, para lograr la saturación categorial, y iii) texto que se presenta en discusión iniciando así la contrastación cualitativa que valida los hallazgos obtenidos.

A manera de ilustración que ejemplifique lo manifestado en esta etapa, en el Cuadro 5 se expone una parte de una matriz cualitativa mientras que la Figura 1 muestra uno de los mapas de conexiones.

\section{¿Qué SE CONCLUYE?}

\section{LA REFLEXIÓN SOBRE LA EXPERIENCIA INVESTIGATIVA}

La experiencia realizada permite destacar diferentes aspectos en correspondencia con el objeto de estudio y la perspectiva investigativa del mismo; así, se pudo corroborar que son factibles de asumir como razones que justifican una RAD: i) el diseño de investigación, particularmente el problema; ii) la visión del grupo investigador de cara al abordaje del problema y iii) el compromiso de análisis e interpretación previo. 
REVISIÓN Y ANÁLISIS DOCUMENTAL PARA ESTADO DEL ARTE:...

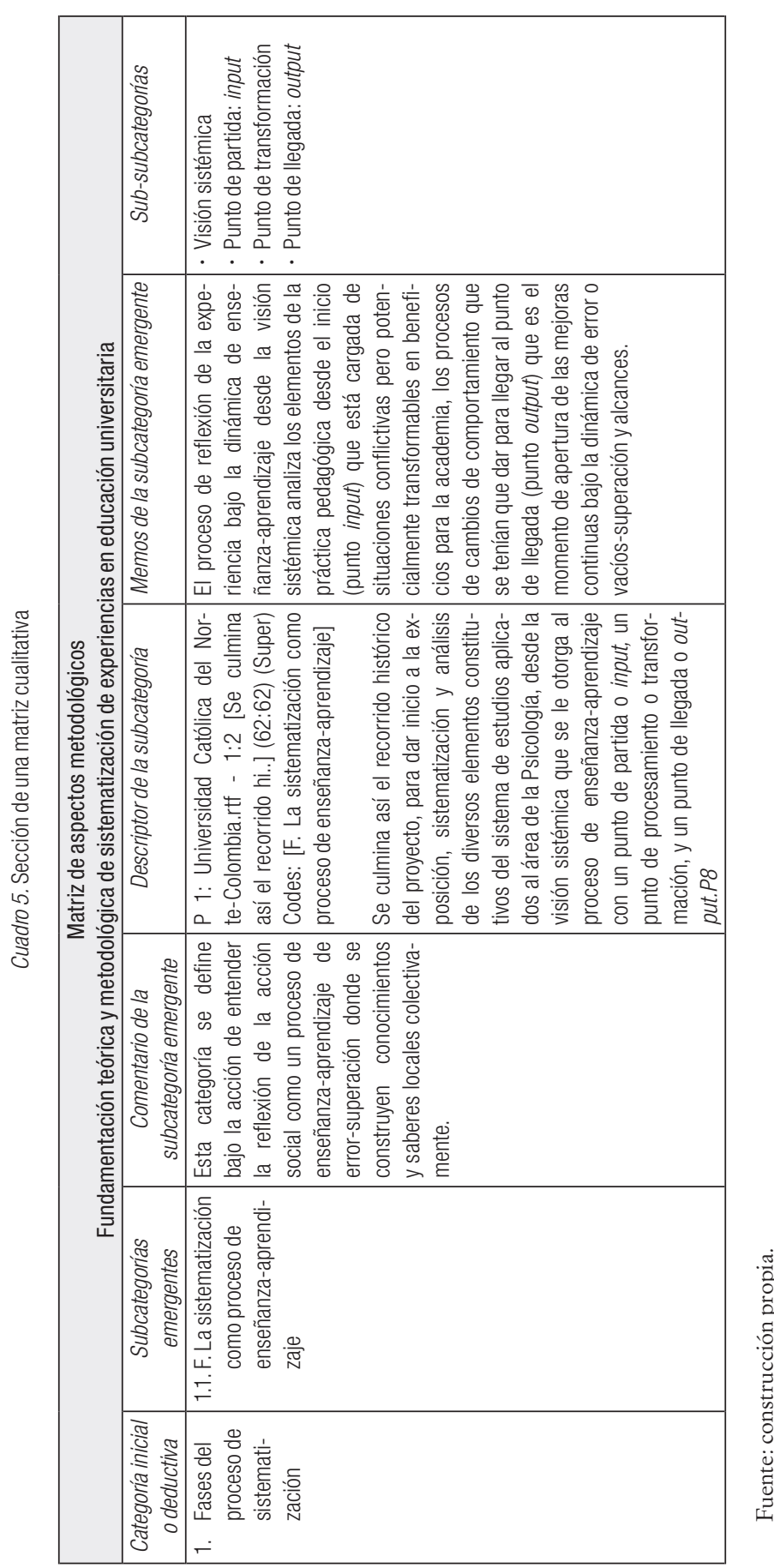




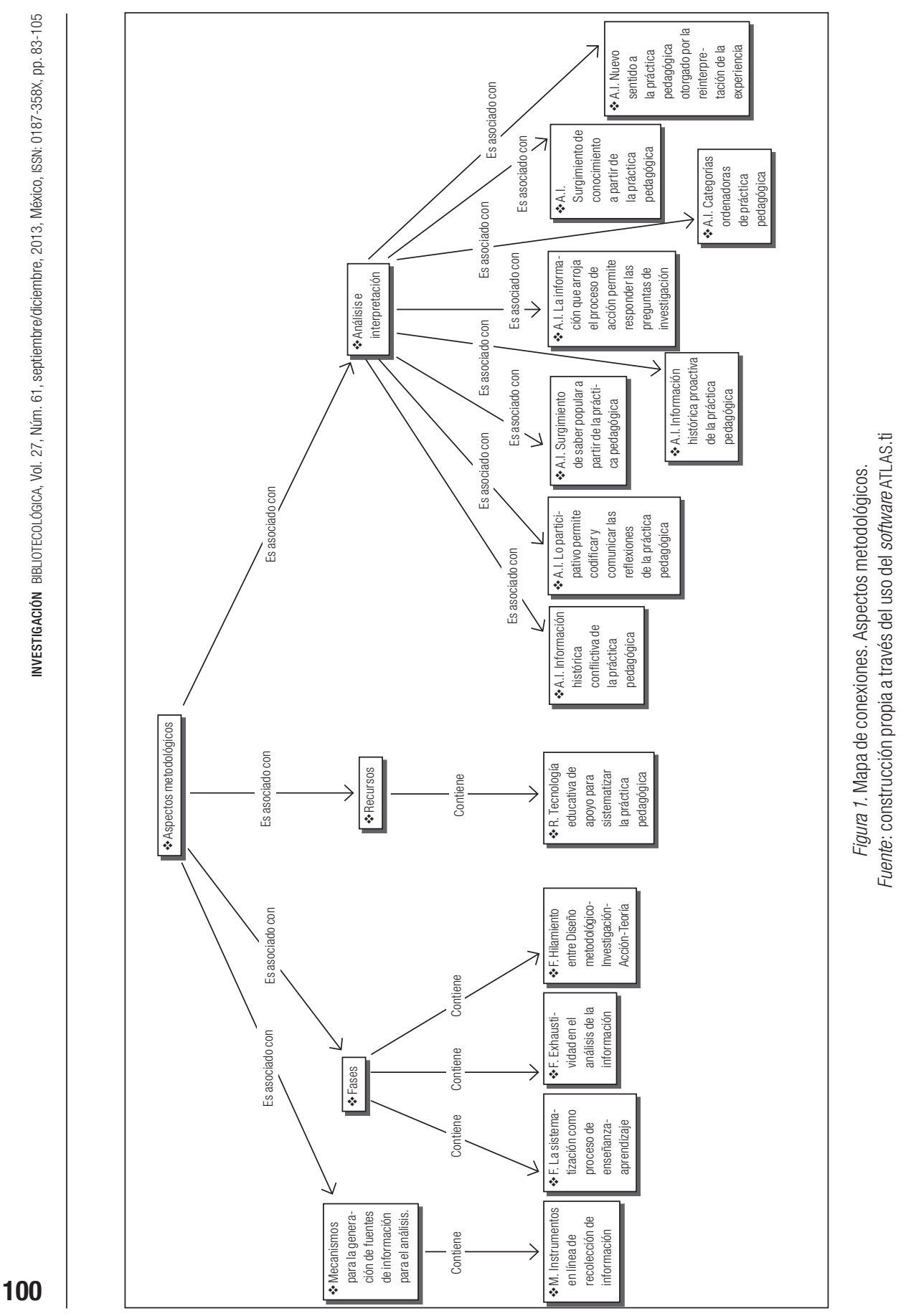


Analizar, apropiar, aplicar y contextualizar referentes teóricos y procedimentales sobre estado del arte y revisión sistemática de literatura se constituyen como bases sólidas para la generación de una metodología de RAD.

Como instrumento de protocolos, el diseño y aplicación de una guía de fase heurística permite cumplir con el propósito específico de definir los parámetros más importantes a tener en cuenta en los procesos de búsqueda y revisión (razones de búsqueda, forma de revisión y evaluación) de las fuentes de información.

Como estrategia de extracción de datos, el diseño y aplicación de un formato de revisión documental es una forma directa de enfocar y favorecer la labor de revisión de fuentes de información, en función de los tópicos relevantes de investigación (el problema y sus tópicos de consulta).

Se considera que el método diseñado: i)favorece el tratamiento sistemático de fuentes de información y resulta beneficioso para el trabajo colectivo; aspecto que, para el caso en particular, tiene correspondencia con la dinámica de la estrategia de seguimiento y observación; ii) constituye una buena herramienta para mejorar, incentivar y obtener resultados concretos de investigación y iii) permite incorporar una modalidad, con cierto grado de sistematización, a las alternativas ya existentes para proyectos de naturaleza análoga.

La metodología de RAD planteada para el estado del arte puede utilizarse como guía para obtener la base que soporte la intervención (resignificación y fortalecimiento) sobre cualquier estrategia de seguimiento y observación de experiencias educativas, pero también para obtener un cimiento para la proyección de nuevos retos investigativos.

Se espera que la experiencia contribuya a continuar el diálogo con los interesados en el problema, con el objeto de contar con mayores elementos en torno a la pertinencia de esfuerzos en materia de metodologías óptimas de RAD para estado del arte.

\section{REFERENCIAS}

Agudelo Cely, Nubia Cecilia; Santana, Martha Yaneth y Velandia, Carmen Cecilia (2005), "Investigación sobre la propia investigación: entretejiendo modelo conceptual y solución informática, en un estado del arte para un programa de educación superior", en Revista de Historia de la Educación Latinoamericana/Rhec, vol. 8, pp. 189-207.

Arellano Sánchez, José y Santoyo Rodríguez, Margarita (2012), La originalidad de la Investigación en torno al Estado del Arte, ponencia presentada en el II Encuentro Latinoamericano de Metodología de las Ciencias Sociales (ELMeCS), agosto, Manizales: Colombia. 
Barbosa Chacón, Jorge Winston; Barbosa Herrera, Juan Carlos; Marciales Vivas, Gloria Patricia y Castañeda-Peña, Harold (2010), "Reconceptualización sobre las competencias informacionales. Una experiencia en la Educación Superior", en Revista de Estudios Sociales, vol. 37, pp. 121-142.

Barbosa Herrera, Juan Carlos (2010), El Guión de Aprendizaje como eje para la virtualización, Proyecto Innova CESAL, casos: Económico Administrativas, Pensamiento complejo y competencias, disponible en: http://www.innovacesal.org/innova_public/cajon_ infos/muestra_informacion_texto_caso [Consultado: 12-06-12].

Barbosa Herrera, Juan Carlos; Rodríguez Villabona, Margarita y Barbosa Chacón, Jorge Winston (2010), Action Research in Higher Education with ICT Incorporation. One way of assessing and transforming the educational proposals, ponencia presentada en International Conference on Education and New Learning Technologies (EDULEARN10), julio, Barcelona, España.

Bauman, Zygmunt (1999), Modernidad Liquida, Buenos Aires: Fondo de Cultura Económica.

Bosh, Mela (s.f.), Entre la heurística y la hermenéutica: las competencias $y$ actitud de los profesionales de la información para la web emergente, disponible en: http://www.webalice.it/melabosch/Contenidos/ Publicaciones/BoschMelaHeurYHermenWebEmergente. pdf [Consultado: 27-11-12].

Bruner, Jerome Seymour (1988), Realidad mental y mundos posibles, Barcelona: Gedisa.

Budgen, David y Brereton, Pearl (2006), Performing Systematic Literature Reviews in Software Engineering, ponencia presentada en el 28th International Conference on Software Engineering, Shanghai, China.

Bucheli Sandoval, Julián Eduardo y Córdoba Lozada, Didier Rubén (s.f.), Estado del arte en el uso de herramientas tradicionales de marketing en las empresas de los sectores: Comercial y de Servicios de la ciudad de Popayán, Cauca, Corporación Universitaria Autónoma del Cauca, disponible en: http://www.ascolfa.edu.co/archivos/ 2.8\%20M\%20-\%20BUCHELI\%20y\%20CORDOBA\%20(P).pdf [Consultado: 27-11-12].

Caro Gutiérrez, María Angélica; Rodríguez Ríos, Alfonso; Calero, Coral; Fernández-Medina, Eduardo y Piattini, Mario (2005), "Análisis y revisión de la literatura en el contexto de proyectos de fin de carrera: una propuesta", en Revista Sociedad Chilena de Ciencia de la Computación, vol. 6, núm. 1, disponible en http:// www.dcc.uchile.cl/\%7Emmarin/revista-sccc/sccc-web/Vol6/CCE SC08.pdf [Consultado: 17-09-12].

Carro, Sandra (2008), "Investigar en la Complejidad. La investigación-acción como propuesta”, en Revista Quebacer Educativo, vol. 92, pp. 66-69, disponible en: http://www.quehacereducativo.edu. uy/docs/6d086b34_92_016\%20rural\%201.pdf [Consultado: 16$10-12]$. 
Castañeda Zapata, Delio Ignacio (2004), "Estado del arte en el aprendizaje organizacional, a partir de las investigaciones realizadas en facultades de Psicología, Ingeniería Industrial y Administración de Empresas en Bogotá, entre los años 1992 y 2002”, en Revista Acta Colombiana de Psicología, vol. 11, pp. 22-33.

De Kerckove, Derrick (1999), Inteligencias en conexión. Hacia una sociedad de la web, Barcelona: Gedisa.

De la Cuesta Benjumea, Carmen (2006), “Teoría y Método. La teoría fundamentada como herramienta de análisis", en Revista Cultura de los Cuidados, vol. 20, pp. 136-140, disponible en http://rua.ua. es/dspace/bitstream/10045/876/1/culturacuidados_20_19.pdf [Consultado: 15-11-12].

Delgado Abella, Leonor Emilia y Forero Aponte, Carlos (2004), "Estado del arte de las investigaciones sobre factores psicológicos en la cultura organizacional, realizadas en facultades de psicología de Bogotá adscritas a ASCOFAPSI (1998 -2003)", en Revista Acta Colombiana de Psicología, vol. 11, pp. 81-96.

Duarte, Jakeline; Zapata, Leidy Patricia y Rentería, Rubiela (2010), “Familia y Primer Infancia: un estado del arte. 1994-2005”, en Revista Estudios Pedagógicos, vol. 36, núm. 1, pp. 107-116.

Fernández Sierra, Myriam (2008), "El proceso investigativo en el estado del arte de la Maestría en Educación de la Universidad Santo Tomás”, en Magistro, vol. 2, núm. 3, pp. 107-112.

García-Valcárcel Muñoz-Repiso, Ana y Tejedor Tejedor, Francisco Javier (2010), "Evaluación de procesos de innovación escolar basados en el uso de las TIC desarrollados en la Comunidad de Castilla y León”, en Revista de Educación, vol. 352, pp. 125-147, disponible en: http://www.revistaeducacion.educacion.es/re352/re352_06.pdf [Consultado: 30-11-12].

Ghiso, Alfredo Manuel (2008), "La Sistematización en contextos formativos universitarios", en Revista Internacional Magisterio. Educación y Pedagogía, vol. 33, pp. 76-79.

Gisbert, Javier y Bonfill, Xavier (2004), “¿Cómo realizar, evaluar y utilizar revisiones sistemáticas y metaanálisis?”, en Revista Gastroenterol Hepatol, vol. 27, núm. 3, pp. 129-149.

Glaser, Barney y Strauss, Anselm (1967), The discovering of grounded theory, New York: Aldine.

Guk, Lju y Kellogg, David (2007), "The ZPD and whole class teaching: Teacher-led and student-led interactional mediation of tasks”, en Language Teaching Research, vol. 11, núm. 3, pp. 281-299.

Gutiérrez Loaiza, Alderid (2012), "Negociaciones de paz en Colombia, 1982-2009. Un estado del arte”, en Revista Estudios Políticos, vol. 40, pp. 175-200.

Hoyos Botero, Consuelo (2000), Un modelo para la investigación documental. Guía Teórico-Práctica sobre Construcción de Estados del Arte con importantes reflexiones sobre la investigación, Medellín: Señal Editora. 
Jiménez Pinto, Juana del Carmen y Calzadilla Muñoz, María Eugenia (2011), "Construcción y Aulas Virtuales: impacto en el proceso de formación docente”, en Revista Apertura, vol. 3, núm. 1, Innovación Educativa.

Kitchenham, Bárbara (2004), Procedures for Performing Systematic Reviews, Keele University Technical Report TR/SE-0401, disponible en: http://tests-zingarelli.googlecode.com/svn-history/r336/trunk/ 2-Disciplinas/MetodPesquisa/kitchenham_2004.pdf [Consultado: 10-11-12]

Lévy, Pierre (1999), ¿Qué es lo virtual?, Barcelona: Paidós.

Lévy, Pierre (2004), Inteligencia colectiva, Washington: Organización Panamericana de la Salud, disponible en: http://inteligenciacolectiva.bvsalud.org [Consultado: 21-09-12].

Londoño, Félix G. (2007), "Semilleros de investigación", en Revista Universidad Eafit, vol. 43, núm. 146, pp. 5-6.

Lopera Quiroz, Olga Lucía y Adarve Calle, Lina (2008), “¿Hay otras maneras de leer la Ciencia?”, en Revista Estudios de Derecho, vol. LXV, núm. 146, disponible en http://aprendeenlinea.udea.edu. co/revistas/index.php/red/article/view/2401/1954 [Consultado: 27-11-12].

Mejía Jiménez, Marco Raúl (2008), La Sistematización. Empodera y produce saber y conocimiento, Bogotá: Ediciones Desde Abajo.

Morín, Edgar (1999), Los siete saberes necesarios para la educación del futuro, París: UNESCO.

Padilla Partida, Siria (2006), "Gestión de ambientes de aprendizaje constructivistas apoyados en la zona de desarrollo próximo", en Revista Apertura, vol. 6, núm. 005, pp. 8-21.

Pantoja Villarreal, María Inés (2006), "Construyendo el objeto de estudio e investigando lo investigado: aplicaciones de un estado del arte”, en Revista Memorias 8, disponible en http://www.revistame morias.com/edicionesAnteriores/8/aplicacionesestadoarte-contad mon.pdf [Consultado: 14-11-12].

Pérez Alcalá, María del Socorro (2009), "La comunicación y la interacción en contextos virtuales de aprendizaje”, en Revista Apertura, vol. 1, núm. 1 [Consultado: 12-10-12].

Pontual Falcao, Taciana y Price, Sara (2010), Interfering and resolving: How tabletop interaction facilitates co-construction of argumentative knowledge, International Society of the Learning Sciences, Inc. [Consultado: 14-10-12].

Posada-González, Nubia (2011), "Aplicabilidad del estado del arte de Carlos Cardona Pescador en Filosofía, Antropología, Ética y Bioética”, en Revista Persona y Bioética, vol. 15, núm. 1, pp. 67-77, disponible en: http://personaybioetica.unisabana.edu.co/index. php/personaybioetica/article/view/1911 [Consultado: 27-11-12].

Postman, Neil (1996), Tecnópolis: la rendición de la cultura a la tecnología, Barcelona: Círculo de Lectores. 
Restrepo Mesa, María Consuelo y Tabares Idárraga, Luis Enrique (2000), "Métodos de Investigación en Educación”, en Revista de Ciencias Humanas, vol. 21, disponible en: http://www.utp.edu. co/ chumanas/revistas/revistas/rev21/restrepo.htm [Consultado: 21-09-12].

Rojas Rojas, Sandra Patricia (2007), "El estado del arte como estrategia de formación en la investigación”, en Revista Studiositas, vol. 2, núm. 3, pp. 5-25, disponible en: http://portalweb.ucatolica.edu. co/easyWeb2/files/1_64_el-estado-del-arte.pdf [Consultado: 1111-12].

Ruiz Botero, L. D. (2001), La sistematización de prácticas, Liceo Nacional Marco Fidel Suárez, Colombia, OEI, disponible en: http:// www.oei.es/equidad/liceo.PDF [Consultado: 04-11-12].

Sígales, Carles (2004) "Formación Universitaria y TIC: nuevos usos y nuevos roles", en Revista Universidad y Sociedad del Conocimiento, vol. 1, núm. 1, disponible en http://www.uoc.edu/rusc/dt/esp/sigales0704.html [Consultado: 29-11-12].

Strauss, Anselm Leonard y Corbin, Juliet (2002), Bases de la investigación cualitativa. Técnicas y procedimientos para desarrollar teoría fundamentada, Medellín: Editorial Universidad de Antioquia.

Uribe Tirado, Alejandro (2004), Acceso, conocimiento y uso de las berramientas especializadas de internet entre la comunidad académica, cientifica, profesional y cultural de la Universidad de Antioquia. Etapa 1: Creación del modelo para recopilación y análisis de información, disponible en: http://docencia.udea.edu.co/investigacioninternet/contenido/depuracion.pdf [Consultado: 20-09-12].

Vélez, Olga Lucía y Galeano, María Eumelia (2002), Estado del arte sobre fuentes documentales en investigación cualitativa, Medellín: Centro de Investigaciones Sociales y Humanas, Universidad de Antioquia.

Wenger, Etienne; McDermott, Richard y Snyder, William (2002), Cultivating communities of practice, Boston: Harvard Business School Press.

Wertsch, James (1988), Vygotsky y la formación social de la mente, Barcelona: Paidós.

Youssef, Adel Ben y Dahmani, Mounir (2008), "The Impact of ICT on Student Performance in Higher Education: Direct Effects, Indirect Effects and Organisational Change”, en Revista de Universidady Sociedad del conocimiento, vol. 5, núm. 1, pp. 45-56. 